\title{
The Type 7 Serotonin Receptor, 5-HT 7 , Is Essential in the Mammary Gland for Regulation of Mammary Epithelial Structure and Function
}

\author{
Vaibhav P. Pai, ${ }^{1,2}$ Laura L. Hernandez, ${ }^{1,3}$ Malinda A. Stull, ${ }^{4}$ and Nelson D. Horseman ${ }^{1}$ \\ ${ }^{1}$ Department of Molecular and Cellular Physiology, University of Cincinnati, Cincinnati, OH 45267, USA \\ ${ }^{2}$ Center for Regenerative and Developmental Biology, Tufts University, Medford, MA 02155, USA \\ ${ }^{3}$ Department of Dairy Science, University of Wisconsin-Madison, Madison, WI 53706, USA \\ ${ }^{4}$ Department of Natural Sciences, Asbury University, Wilmore, KY 40390, USA
}

Correspondence should be addressed to Vaibhav P. Pai; vaibhav.pai@tufts.edu and Nelson D. Horseman; horsemn@ucmail.uc.edu

Received 10 September 2014; Revised 19 December 2014; Accepted 21 December 2014

Academic Editor: Yun Yen

Copyright (C) 2015 Vaibhav P. Pai et al. This is an open access article distributed under the Creative Commons Attribution License, which permits unrestricted use, distribution, and reproduction in any medium, provided the original work is properly cited.

\begin{abstract}
Autocrine-paracrine activity of serotonin (5-hydroxytryptamine, 5-HT) is a crucial homeostatic parameter in mammary gland development during lactation and involution. Published studies suggested that the 5- $\mathrm{HT}_{7}$ receptor type was important for mediating several effects of 5-HT in the mammary epithelium. Here, using 5- $\mathrm{HT}_{7}$ receptor-null (HT7KO) mice we attempt to understand the role of this receptor in mediating 5-HT actions within the mammary gland. We demonstrate for the first time that HT7KO dams are inefficient at sustaining their pups. Histologically, the HT7KO mammary epithelium shows a significant deviation from the normal secretory epithelium in morphological architecture, reduced secretory vesicles, and numerous multinucleated epithelial cells with atypically displaced nuclei, during lactation. Mammary epithelial cells in HT7KO dams also display an inability to transition from lactation to involution as normally seen by transition from a columnar to a squamous cell configuration, along with alveolar cell apoptosis and cell shedding. Our results show that $5-\mathrm{HT}_{7}$ is required for multiple actions of 5-HT in the mammary glands including core functions that contribute to changes in cell shape and cell turnover, as well as specialized secretory functions. Understanding these actions may provide new interventions to improve lactation performance and treat diseases such as mastitis and breast cancer.
\end{abstract}

\section{Background}

The mammary gland cycles through waves of proliferation and regression associated with each pregnancy $[1,2]$. This unique regenerative ability of the mammary gland is driven by the hormonal surges associated with pregnancy and lactation with each cycle consisting of a burst of alveolar epithelial growth and differentiation during pregnancy, followed by copious alveolar milk production during lactation, and concluding with massive epithelial apoptosis and remodeling during involution. The dynamic nature of the mammary gland also makes it vulnerable to breast diseases like mastitis and breast cancer. Such diseases arise due to the dysfunctioning of fundamental homeostatic regulatory mechanism that governs mammary gland development and function.
A number of recent studies implicate the regressing phase of the mammary gland (involution) in creating conditions facilitative for breast diseases including breast cancer [36]. During mammary gland involution, there is a systematic disassembly of the established lactation machinery, which is triggered by weaning-induced milk accumulation (milk stasis) within the glands and disruption of epithelial barrier function [7]. Local factors are largely implicated in inducing mammary involution [8-10]. One of the local factors synthesized and secreted by mammary epithelial cells is the monoamine serotonin (5-hydroxytryptamine, 5-HT) [11, 12]. 5 -HT is potently induced upon milk stasis and is a crucial autocrine-paracrine regulator of involution. This action of 5HT is conserved through many species [11-14].

Similar to other tissues, the bioactive level of 5-HT in the mammary gland is dynamically regulated by tryptophan hydroxylase (TPH), the 5-HT reuptake transporter (SERT), 
and monoamine oxidase (MaO) [11, 14-16]. 5-HT exerts its actions through 7 classes of receptors $\left(5-\mathrm{HT}_{1-7}\right)$ encoded by 17 human genes [17]. The receptors are G-protein coupled with the exception of the ionotropic type 3 receptors $\left(5-\mathrm{HT}_{3 \mathrm{~A}-\mathrm{E}}\right)$, which are ligand-gated cation channels. The mammary epithelium expresses multiple 5 - $\mathrm{HT}$ receptors [15, $18,19]$, with $5-\mathrm{HT}_{7}$ having been shown to be important, based on in vitro studies $[15,18-20]$. $5-\mathrm{HT}_{7}$ is a $\mathrm{G}_{\mathrm{s}}$-coupled receptor, which induces cAMP accumulation in the mammary epithelial cells upon 5-HT binding $[15,17,18,20]$. 5-HT through $5-\mathrm{HT}_{7}$ regulates mammary epithelial TJ in vitro $[15,20,21]$. However, the process of involution involves multiple events regulated simultaneously in addition to TJ regulation. Here, we use HT7KO mice to gain insights into the other cellular events regulated by $5-\mathrm{HT}$ through $5-\mathrm{HT}_{7}$.

Here, we show that HT7KO dams were inefficient at sustaining their pups, partly due to decrease in milk secretion necessary to sustain their pups. In addition, HT7KO epithelium failed to undergo morphological and physiological changes associated with the transition from lactation to involution. These findings are crucial in understanding the homeostatic mechanism employed by $5-\mathrm{HT}$ in regulating epithelial morphological changes brought forth during transition from lactation to involution and may give possible insights into its role in creating conditions favorable for breast pathologies.

\section{Results}

2.1. HT7KO Dams Fail to Sustain Their Pups. In vitro experiments had been published indicating that $5-\mathrm{HT}_{7}$ receptors are involved in mediating 5-HT actions in the mammary glands $[15,18,20,21]$. To determine the in vivo physiological significance of $5-\mathrm{HT}_{7}$ signaling for mammary gland development, we examined lactation in HT7KO mice. Wild type (WT) and HT7KO female littermates were mated to WT sires and their ability to bear litters and sustain pups was analyzed. There was no difference in the ability of HT7KO mice to get pregnant, gestate, and give birth to live pups. However, it was clear that the offspring of HT7KO mothers died at a high rate during the first few days of life. Because of the potential roles of $5-\mathrm{HT}_{7}$ in the brain, maternal behavior was carefully observed. HT7KO mothers displayed normal maternal behaviors (nesting, crouching, and pup grooming), and the nursing behavior of the pups (nipple attachment and sucking) was also normal.

To determine whether lactation was defective in the HT7KO mothers, WT offspring were cross-fostered onto either WT or HT7KO mothers on day one after birth. WT dams fostered WT pups with $100 \%$ survival during the first week of life. In contrast, $66 \%$ of the WT pups fostered with HT7KO dams died by day 4 after parturition (Figure 1). Each dam was kept with a small litter ( $n=6$ pups) so that the dams are not under much pressure for lactation. Even at such low lactation pressure, the pup survival was affected in the HT7KO suggesting inefficient lactation. Bodies of the dead pups were discovered intact indicating that pup deaths were caused by malnutrition and dehydration and not cannibalism (Figure 1(a)).

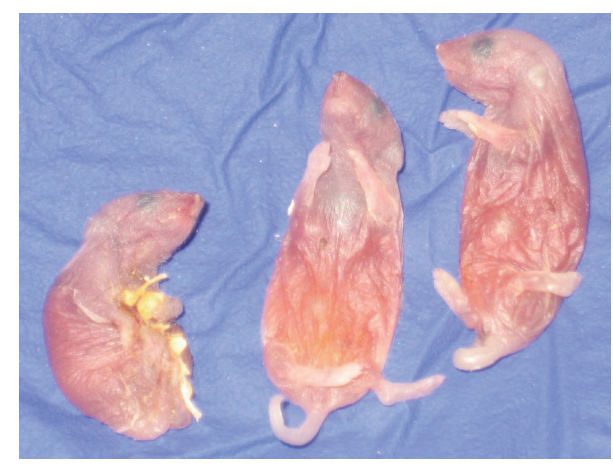

(a)

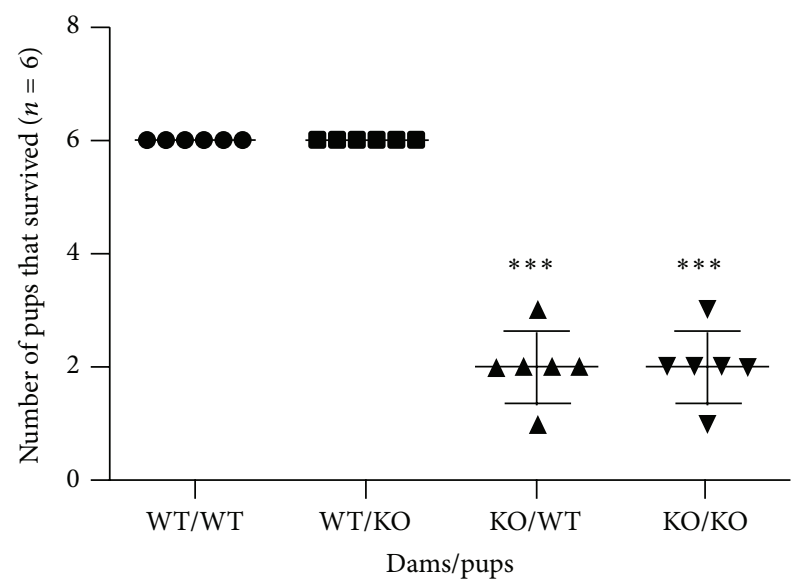

(b)

FIGURE 1: HT7KO dams are inefficient at sustaining their pups. (a) Image of dead WT pups from the HT7KO dam's cross-fostered litter showing the shriveled yet intact bodies of the pups suggesting their death due to insufficient nutrition. (b) This represents a graph of a number of pups (out of $n=6$ ) that survived when fostered with WT or HT7KO dams. $N=6$ dams was maintained for each group. The data was analyzed by two-way ANOVA. ${ }^{* * *} P<0.001$.

\subsection{Epithelial Abnormalities in Lactating HT7KO Glands.} Lactating mammary glands on day 10 postpartum were examined. In order to equalize lactation pressure on the dams, litter sizes were kept at 10 by replacing pups that died or were obviously weak. Histomorphologies of WT and HT7KO mammary glands were compared to explore possible reasons for the observed lactation defects and multiple abnormalities were observed.

The HT7KO epithelium contained numerous bi- and multinucleated cells (Figures 2(a) and 2(b), black arrowheads, $P<0.001)$, which were uncommon in the epithelium of WT type glands. Perhaps most strikingly, the basal positioning of nuclei, characteristic of normal columnar epithelia (Figure 2(a), black arrow), was disrupted in the majority of the HT7KO mammary epithelial cells. Nuclei were inconsistently located in the HT7KO cells, with many apical nuclei (Figure 2(a), black arrowheads), contributing to a scalloped appearance of the luminal epithelial surface. The unusual positioning of nuclei often, but not always, corresponded with multinucleated cells. About $50 \%$ of HT7KO alveolar cells were bi- or multinucleated (Figure 2(b)), and misplaced 

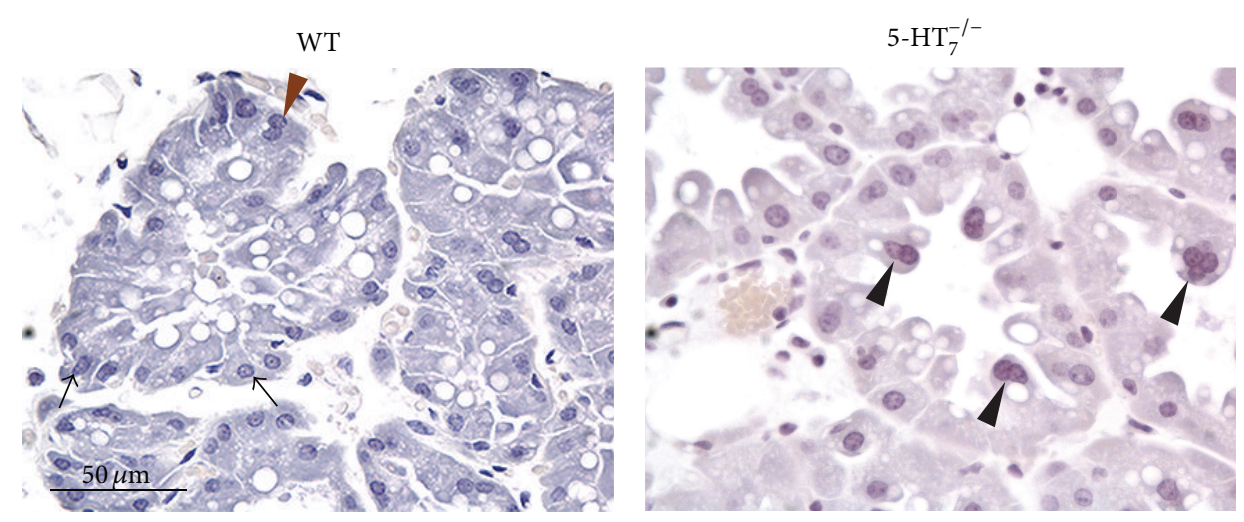

(a)

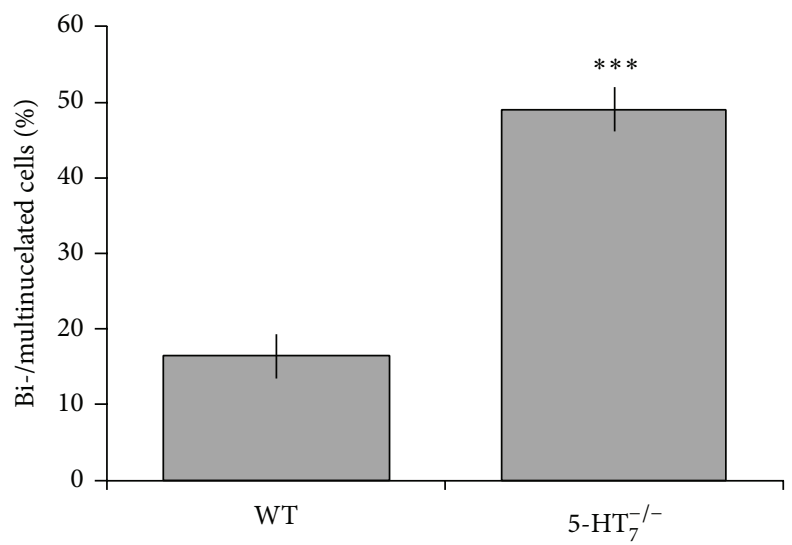

(b)

FIGURE 2: HT7KO lactating epithelium shows dysregulated cellular architecture. (a) WT and HT7KO glands were harvested during peak lactation (day 10) and stained with hematoxylin. Black arrows indicate mononucleated cells with basal nuclear localization. Brown arrowhead indicates binucleated cells with basal nuclear localization. Black arrowheads indicate the bi- and multinucleated cells that have nonbasal nuclear localizations. (b) Quantitative analysis of the multinucleated cells represented as \% of alveolar cells that are bi-/multinucleated per alveolus. The sections of glands from various mice were counted. The data is represented as mean \pm SEM. The data was analyzed using $t$-test. ${ }^{* * *} P<0.001$.

nuclei ranged from 28 to $60 \%$ in different alveoli. The luminal epithelial surface in lactating mammary glands is typically very dynamic, fimbriated, and populated by numerous lipid globules and secretory droplets. The luminal surfaces of 5HT7KO glands appeared to be congested by comparison, consistent with the poor lactational performance of these dams. These observations suggested that $5-\mathrm{HT}_{7}$ has important cellular functions that are essential for normal epithelial homeostasis within the lactating mammary glands.

2.3. Response of HT7KO Epithelium to Milk Stasis. Glands of lactating dams were subjected to unilateral teat-sealing as a way of challenging epithelial homeostasis and establishing the participation of local regulatory mechanisms. Representative images from WT and HT7KO glands are depicted in Figure 3(a). Note that these sections were stained more deeply than those in Figure 2 so as to visualize structures in the sealed glands, which are less basophilic and therefore do not take stain as readily as actively lactating glands. WT lactating (WT-open) glands were characterized by tightly packed columnar epithelia lining the secretory alveolar units and the lumens were filled with secretory material (green arrowhead). The epithelial cells were highly basophilic and contained large and numerous secretory vesicles and lipid globules (red arrowheads), consistent with active biosynthetic and secretory activities. Three features that were obviously different from normal lactating epithelium were seen in the HT7KO lactating glands (Figure 3(a), HT7KO, open): (1) the free apical surface was scalloped, with bulbous or rounded tips, (2) the alveolar lumens were largely devoid of stained secretory material, and (3) the epithelial cells lining the alveoli had fewer and smaller vesicles and lipid globules. The other differences were in the nuclei distributions, as shown in Figure 2, which are difficult to see in Figure 3 because of the darker staining.

In response to milk stasis, the WT glands underwent the characteristic changes in morphology that have been documented during milk stasis [2, 22] (Figure 3(a)). The epithelium changed from being highly columnar to flattened (squamous transition) (Figures 3(a) and 3(b)(i)). Large amounts of secreted material (milk) accumulated within the alveolar lumens (Figure 3(a)) with a concomitant increase 

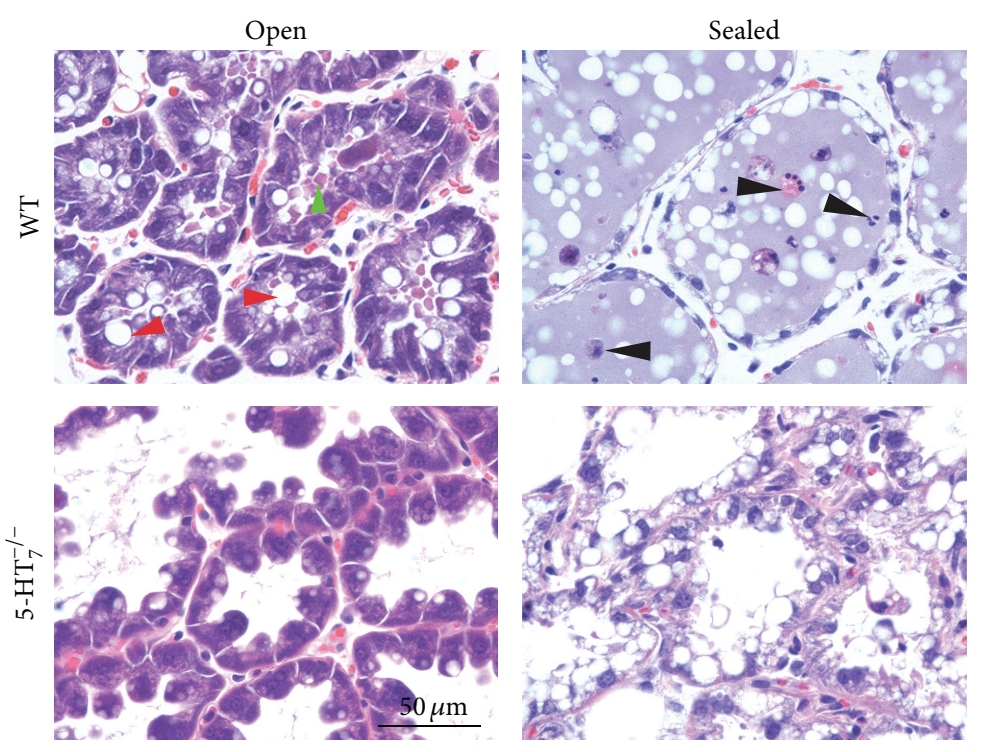

(a)

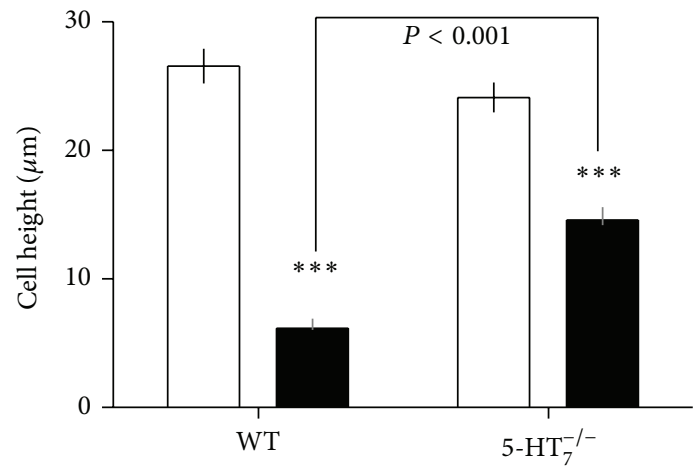

(i)

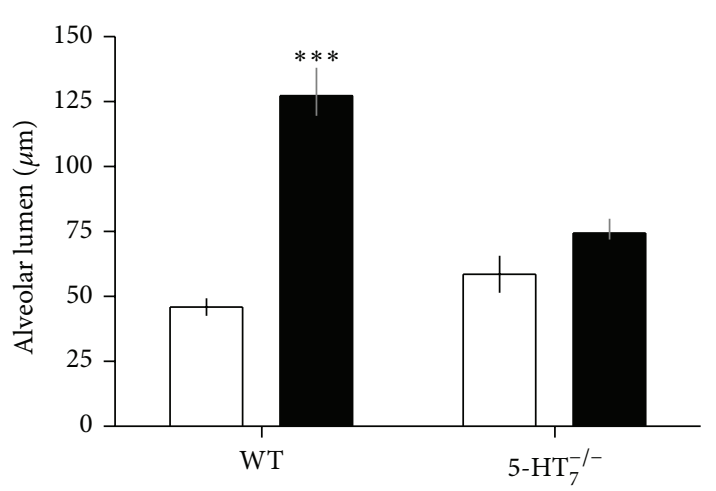

(ii)

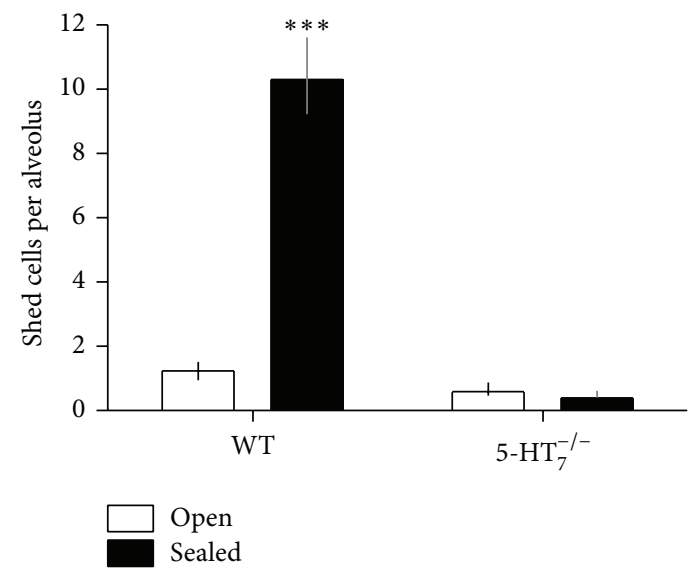

(iii)

(b)

FIGURE 3: Lactating HT7KO mammary glands are morphologically altered and fail to undergo morphological transition associated with milk stasis induced involution. (a) A unilateral teat-sealing experiment was performed on WT and HT7KO lactating mice at peak lactation (day 10). Sealed and unsealed glands were harvested 48 hours (day 2$)$ after sealing $(n=3)$. Representative contralateral (sealed and open) hematoxylin and eosin stained sections from number 4 glands are shown. Red arrowheads point to the secretory vesicles present within the mammary epithelial cells. Green arrowhead indicates secreted materials filling the alveolar lumen. Black arrowheads point to apoptotic shed cells. (b) Morphometric data on the mammary glands with respect to (i) cell height, (ii) alveolar lumen diameters, and (iii) number of shed cells per alveolus. The data was analyzed by two-way ANOVA. ${ }^{*} P<0.05,{ }^{* *} P<0.01$, and ${ }^{* * *} P<0.001$. 
in the alveolar lumen diameters in comparison to lactating glands (Figures 3(a) and 3(b)(ii)). Typically, the lumens contained many shed cells undergoing cell death (black arrowheads, Figures 3(a) and 3(b)(iii)) [23]. In comparison, the sealed HT7KO glands failed to undergo the same set of morphological changes in response to milk stasis. The knockout epithelium failed to completely transition to a squamous configuration (Figures $3(\mathrm{a})$ and $3(\mathrm{~b})(\mathrm{i})$, (ii)). The HT7KO epithelial cells had large vacuoles and/or lipid globules (Figure 3(a)), but the alveolar lumens appeared empty and the luminal spaces were not expanded (Figures 3(a) and 3(b)(ii)). The HT7KO glands did not show any increase in cells being shed into the lumens and undergoing cell death (Figures 3(a) and 3(b)(iii)).

\section{Discussion}

Intramammary 5-HT signaling is crucial for lactation homeostasis and transition into involution $[2,11,12,14-16,24$, 25]. Mammary epithelial cells express a variety of relevant genes that encode the machinery necessary to synthesize, secrete, and respond to 5-HT [11, 14, 15, 18-21]. Mammary epithelial cells express $5-\mathrm{HT}_{1 \mathrm{D}, 2 \mathrm{~B}, 3 \mathrm{~A}}$ and ${ }_{7}$ receptors $[18,19]$.

Studies using pharmacological and in vitro approaches indicated that $5-\mathrm{HT}_{7}$ regulated the epithelial tight junctions in mammary glands [14-16, 20]. To gain further insights into $5-\mathrm{HT}_{7}$ functions at the tissue level, we used mice genetically deficient for $5-\mathrm{HT}_{7}$ (HT7KO) [26] in the studies described here. HT7KO female mice conceived, progressed through pregnancy, and bore pups normally. However, the knockout dams had problems rearing their pups, as $66 \%$ of cross-fostered pups died from what appeared to be malnutrition and dehydration. The lactating HT7KO glands had significantly impaired milk production. In addition, during milk stasis, the HT7KO epithelium appeared to be highly vacuolated with empty alveolar lumens, further suggesting impaired secretion. TPH1 knockout (TPH1KO) mice showed a variety of defects in mammary epithelial homeostasis. When responding to prolactin, the TPH1KO glands were hypersecretory $[11,14]$. Correspondingly, when hormonestimulated glands were in milk stasis, the epithelium failed to transition to the typical squamous morphology. Many fewer cells were shed into the alveolar lumens, and therefore they did not undergo detachment-induced cell death (anoikis) $[22,23,27]$. These mammary phenotypes in TPH1KO mice were similar in many ways to the defects here in the HT7KO mice. An important difference between these models is that TPH1KO mice produced sufficient milk to nurse their litters, whereas HT7KO mothers did not. These differences suggest that unbalanced $5-\mathrm{HT}_{7}$ signaling in the absence of type 7 receptors is detrimental to milk secretion.

A critical cell physiological event in mammary epithelial homeostasis is the opening of tight junctions in response to 5-HT-mediated p38 MAPK signaling $[2,15,16,20]$. Because tight junctions are the only planar cell-cell junctions in the lactating mammary epithelium [28, 29], failure of tight junction disruption in HT7KO glands is sufficient to explain the defects observed during milk stasis.
3.1. $5-\mathrm{HT}_{7}$ Mediates Morphological Transitions of Mammary Epithelium in Response to Milk Stasis. The lactating mammary gland is in a dynamic equilibrium. The production of milk must be balanced with the delivery of the milk to the nursing pups in order for the secretory properties of the gland to be maintained. In the absence of suckling, milk accumulation occurs and the gland is triggered to initiate events that ultimately lead to involution $[2,27]$. Teat-sealing induces milk stasis, precipitating dramatic changes in the epithelial architecture in normal glands. In the epithelium transitions from a columnar to a squamous configuration, there is significant distention of the alveoli and many cells are shed and die. In contrast to normal glands, the HT7KO mammary epithelium failed to undergo the full range of morphological changes associated with milk stasis. The epithelium retained a columnar shape, and cell shedding was greatly reduced. Given that tight junctions are the only cellcell junction in the lactating mammary epithelium, failure to disrupt tight junctions is sufficient to explain the lack of morphological changes and cell shedding. These findings correspond with the previous in vitro observations using selective $5-\mathrm{HT}_{7}$ antagonist treatment. Inhibition of $5-\mathrm{HT}_{7}$ resulted in enhanced epithelial membrane resistance and increased milk protein gene expression [15, 18, 19]. These results suggest that $5-\mathrm{HT}$ acting through $5-\mathrm{HT}_{7}$ is a critical mediator of the morphological and physiological transitions associated with cessation of milk synthesis.

\subsection{5- $\mathrm{HT}_{7}$ Mediates 5-HT Actions of Epithelial Cell Shedding} and Cell Death. A characteristic feature of the involuting mammary gland is a marked increase in cell shedding and associated cell death [2, 27]. This has been proposed as a mechanism to rid the alveoli of spent cells and is also the most expedient way to eliminate cells that have been terminally differentiated as a means to prevent further milk accumulation. Previous studies using an in vitro model of functionally differentiated mammary epithelial cells [30] have shown that 5-HT induces epithelial cell shedding and cell death [21]. Interestingly, sealed HT7KO glands failed to exhibit any increase in epithelial shedding and cell death in comparison to lactating HT7KO glands. This demonstrates that $5-\mathrm{HT}_{7}$ is critically involved in bringing about epithelial cell shedding and death.

A highly regulated cell turnover is critical in maintaining the secretory properties of the terminally differentiated lactating mammary epithelium. At peak lactation (day 10) in normal mammary glands, a small number of binucleated cells were observed. In contrast, the HT7KO lactating glands had dramatically increased numbers of binucleated (and multinucleated) cells (50\%). In addition to the increased number of cells with multiple nuclei, it is important to note the disruption of the basal placement of the nuclei within the cells. These observations demonstrate that loss of 5$\mathrm{HT}_{7}$ results in the dysregulation of cell renewal, not only in the number of cells undergoing nuclear division but also in fundamental differences in the intracellular organization within the cells.

A similar phenotype of multinucleated, highly vacuolated mammary epithelial cells is observed with overexpression 
of the E3 ubiquitin ligase protein MDM2 [31]. MDM2 overexpression in mammary epithelial cells resulted in the uncoupling of S-phase from mitosis, causing multiple rounds of DNA synthesis without cell division. Also, MDM2 is closely associated with suppression of cell death through inactivation of p53 tumor suppressor [31-33]. Interestingly, MDM2 expression is suppressed by increased intracellular cAMP levels [32]. 5- $\mathrm{HT}_{7}$ activation results in increased intracellular cAMP in the mammary epithelial cells $[15,20]$. This in turn may suppress MDM2. Conversely, the absence of $5-\mathrm{HT}_{7}$ activation, as is the case in $5-\mathrm{HT}_{7}^{-1-}$ mice, may result in sustained increases of MDM2 and increased cell survival. This dysregulation of MDM2 expression may then be a first step towards transformation and neoplastic growth within the mammary epithelium. This is consistent with other observations where MDM2 overexpression is linked to breast tumors and hyperplastic alveolar nodules in mice and also human breast cancers $[31,34]$. The activation of $5-\mathrm{HT}_{7}$, therefore, may serve as a deterrent against transformation of mammary epithelial cells. This hypothesis is supported by our previous observations on the complex role of 5-HT in breast cancers [18]. Our in vitro observation of $5-\mathrm{HT}_{7}$ receptormediated suppression of cell growth/division lends further support to this hypothesis [18]. In addition, absence of 5- $\mathrm{HT}_{7}$ could result in enhanced activation of the $5-\mathrm{HT}_{2 \mathrm{~B}}$ receptor, which has been shown to have proliferative effects in other organ systems $[35,36]$. Hence, it is conceivable that a lack of 5-HT signal through 5- $\mathrm{HT}_{7}$ (growth inhibitory, cell shedding, and cell death) leads to a shift in the balance of epithelial turnover, resulting in a multinucleated, overproliferating, and nonshedding epithelium.

\section{Conclusions}

In conclusion, the studies reported here provide valuable insights into the functions of $5-\mathrm{HT}_{7}$ receptor signal in mediating various actions of 5-HT. Through the use of HT7KO mice, we demonstrate that the contribution of this receptor is crucial in maintaining the lactation capacity of the mammary gland. The $5-\mathrm{HT}_{7}$ receptor is involved in regulating epithelial cell growth, shedding, and cell death. The regulation of epithelial tight junctions is likely to be the mechanistic common denominator in these phenotypes. Understanding how $5-\mathrm{HT}_{7}$ downstream signals are able to regulate many crucial functions will be critical in making inroads towards achieving better regulation of mammary gland development, lactation, nursing, and understanding its involvement in breast pathologies including breast cancer.

\section{Materials and Methods}

5.1. Animal Studies. HT7KO mice (C57BL/6J background) were obtained from The Scripps Research Institute (Hedlund et al.) [26]. Mice were maintained on a L, D:14, 10 daily light, darkness cycle with ad libitum food and water. Female HT7KO mice obtained by heterozygous crosses were used during lactation. Wild type (defined as homozygous $+/+$ and heterozygous $+/-$ ) littermates were phenotypically normal and were used as controls. To perform teat-sealing experiments, mice upon reaching peak lactation (day 10) were lightly anesthetized by isoflurane inhalation. The nipples on the left side of the body were exteriorized and sealed using a suture and surgical glue. The glands on the right side of the body remained open to serve as contralateral lactation controls $[11,22]$. The dams were returned to their pups and visual confirmation of pups resuming suckling was obtained. Pups were allowed to nurse for $48 \mathrm{hrs}$ after teat-sealing, at which time the dams were sacrificed using $\mathrm{CO}_{2}$ inhalation followed by cervical dislocation and the glands harvested and stored in $4 \%$ paraformaldehyde at $4^{\circ} \mathrm{C}$ overnight, then placed in $70 \%$ ethanol, and processed for tissue sectioning and hematoxylin and eosin staining.

5.2. Immunostaining. Mammary glands were harvested and fixed in $4 \%$ paraformaldehyde, followed by paraffin embedding. The samples were sectioned at $4 \mu \mathrm{m}$ and used for staining. The sections were permeabilized in $0.1 \%$ Triton-X100. The following stains were used: hematoxylin and eosin. Images were collected using an Olympus Microscope.

5.3. Data Analysis. Data analysis was conducted using Prism, version 5.0b (GraphPad Software, San Diego, CA). An $n=6$ was maintained for mice in each group, unless otherwise mentioned. All graphed results are represented as mean \pm SEM. One-way ANOVA with Tukey's post hoc test or twoway ANOVA with Bonferroni's post hoc test was used to analyze the data. A probability level of 0.05 was considered as significant. ${ }^{*} P<0.05,{ }^{* *} P<0.01$, and ${ }^{* * *} P<0.001$.

5.4. Ethics Statement. All experiments were conducted under protocols that were approved (\#05-01-11-01) by the Institutional Animal Care and Use Committee at University of Cincinnati.

\section{Abbreviations \\ 5-HT: 5-hydroxytryptamine, serotonin \\ $5-\mathrm{HT}_{7}$ : Type 7 serotonin receptor protein \\ SERT: Serotonin reuptake transporter \\ TPH: Tryptophan hydroxylase \\ TJ: Tight junctions.}

\section{Conflict of Interests}

The authors declare that there is no conflict of interests regarding the publication of this paper.

\section{Authors' Contribution}

Vaibhav P. Pai, Laura L. Hernandez, and Nelson D. Horseman designed the research, Vaibhav P. Pai and Laura L. Hernandez performed the research, and provided technical and research support. Nelson D. Horseman, Malinda A. Stull, and Vaibhav P. Pai wrote the paper. All the authors read and approved the paper. 


\section{Acknowledgments}

This project was supported by National Research Initiative Competitive Grant no. 2007-35206-17898 from the USDA Cooperative State Research, Education and Extension Service. The funders had no role in study design, data collection and analysis, decision to publish, or preparation of the paper. The authors thank Dr. Peter Dedlund for the generous donation of $5-\mathrm{HT}_{7}$ receptor knock-out mice for this study.

\section{References}

[1] M. T. Lewis, "Homeobox genes in mammary gland development and neoplasia," Breast Cancer Research, vol. 2, no. 3, pp. 158-169, 2000.

[2] V. P. Pai and N. D. Horseman, "Mammary gland involution: events, regulation and influences on breast disease," in Endothelium and Epithelium: Composition, Functions and Pathology, J. Carrasco and M. Mota, Eds., Human Anatomy and Physiology: Cell Biology Research Progress, p. 247, NOVA Science Publishers, New York, NY, USA, 2011.

[3] K. Polyak, "Pregnancy and breast cancer: the other side of the coin," Cancer Cell, vol. 9, no. 3, pp. 151-153, 2006.

[4] P. Schedin, "Pregnancy-associated breast cancer and metastasis," Nature Reviews Cancer, vol. 6, no. 4, pp. 281-291, 2006.

[5] G. Albrektsen, I. Heuch, S. Tretli, and G. Kvale, "Breast cancer incidence before age 55 in relation to parity and age at first and last births: a prospective study of one million Norwegian women," Epidemiology, vol. 5, no. 6, pp. 604-611, 1994.

[6] G. Albrektsen, I. Heuch, G. Kvale et al., "Further evidence of a dual effect of a completed pregnancy on breast cancer risk," Cancer Causes and Control, vol. 7, no. 4, pp. 487-488, 1996.

[7] C. M. Alexander, S. Selvarajan, J. Mudgett, and Z. Werbc, "Stromelysin-1 regulates adipogenesis during mammary gland involution," Journal of Cell Biology, vol. 152, no. 4, pp. 693-703, 2001.

[8] C. J. Wilde, D. T. Calvert, A. Daly, and M. Peaker, "The effect of goat milk fractions on synthesis of milk constituents by rabbit mammary explants and on milk yield in vivo. Evidence for autocrine control of milk secretion," Biochemical Journal, vol. 242, no. 1, pp. 285-288, 1987.

[9] K. A. K. Hendry, K. J. Simpson, K. R. Nicholas, and C. J. Wilde, "Autocrine inhibition of milk secretion in the lactating tammar wallaby (Macropus eugenii)," Journal of Molecular Endocrinology, vol. 21, no. 2, pp. 169-177, 1998.

[10] C. H. Knight, M. Peaker, and C. J. Wilde, "Local control of mammary development and function," Reviews of Reproduction, vol. 3, no. 2, pp. 104-112, 1998.

[11] M. Matsuda, T. Imaoka, A. J. Vomachka et al., "Serotonin regulates mammary gland development via an autocrine-paracrine loop," Developmental Cell, vol. 6, no. 2, pp. 193-203, 2004.

[12] L. L. Hernandez, C. M. Stiening, J. B. Wheelock, L. H. Baumgard, A. M. Parkhurst, and R. J. Collier, "Evaluation of serotonin as a feedback inhibitor of lactation in the bovine," Journal of Dairy Science, vol. 91, no. 5, pp. 1834-1844, 2008.

[13] D. L. Hadsell, A. F. Parlow, D. Torres, J. George, and W. Olea, "Enhancement of maternal lactation performance during prolonged lactation in the mouse by mouse GH and long-R3IGF-I is linked to changes in mammary signaling and gene expression," Journal of Endocrinology, vol. 198, no. 1, pp. 61-70, 2008.
[14] A. M. Marshall, L. A. Nommsen-Rivers, L. L. Hernandez et al., "Serotonin transport and metabolism in the mammary gland modulates secretory activation and involution," The Journal of Clinical Endocrinology and Metabolism, vol. 95, no. 2, pp. 837846, 2010.

[15] M. A. Stull, V. Pai, A. J. Vomachka, A. M. Marshall, G. A. Jacob, and N. D. Horseman, "Mammary gland homeostasis employs serotonergic regulation of epithelial tight junctions," Proceedings of the National Academy of Sciences of the United States of America, vol. 104, no. 42, pp. 16708-16713, 2007.

[16] L. L. Hernandez, J. L. Collier, A. J. Vomachka, R. J. Collier, and N. D. Horseman, "Suppression of lactation and acceleration of involution in the bovine mammary gland by a selective serotonin reuptake inhibitor," Journal of Endocrinology, vol. 209, no. 1, pp. 45-54, 2011.

[17] J. R. Raymond, Y. V. Mukhin, A. Gelasco et al., "Multiplicity of mechanisms of serotonin receptor signal transduction," Pharmacology \& Therapeutics, vol. 92, no. 2-3, pp. 179-212, 2001.

[18] V. P. Pai, A. M. Marshall, L. L. Hernandez, A. R. Buckley, and N. D. Horseman, "Altered serotonin physiology in human breast cancers favors paradoxical growth and cell survival," Breast Cancer Research, vol. 11, no. 6, article R81, 2009.

[19] L. L. Hernandez, S. W. Limesand, J. L. Collier, N. D. Horseman, and R. J. Collier, "The bovine mammary gland expresses multiple functional isoforms of serotonin receptors," Journal of Endocrinology, vol. 203, no. 1, pp. 123-131, 2009.

[20] V. P. Pai and N. D. Horseman, "Biphasic regulation of mammary epithelial resistance by serotonin through activation of multiple pathways," The Journal of Biological Chemistry, vol. 283, no. 45, pp. 30901-30910, 2008.

[21] V. P. Pai and N. D. Horseman, "Multiple cellular responses to serotonin contribute to epithelial homeostasis," PLOS ONE, vol. 6, no. 2, Article ID e17028, 2011.

[22] M. Li, X. Liu, G. Ribinson et al., "Mammary-derived signals activate programmed cell death during the first stage of mammary gland involution," Proceedings of the National Academy of Sciences of the United States of America, vol. 94, no. 7, pp. 34253430, 1997.

[23] J. Monks, C. Smith-Steinhart, E. R. Kruk, V. A. Fadok, and P. M. Henson, "Epithelial cells remove apoptotic epithelial cells during post-lactation involution of the mouse mammary gland," Biology of Reproduction, vol. 78, no. 4, pp. 586-594, 2008.

[24] S. Iden and J. G. Collard, "Crosstalk between small GTPases and polarity proteins in cell polarization," Nature Reviews Molecular Cell Biology, vol. 9, no. 11, pp. 846-859, 2008.

[25] R. J. Collier, L. L. Hernandez, and N. D. Horseman, "Serotonin as a homeostatic regulator of lactation," Domestic Animal Endocrinology, vol. 43, no. 2, pp. 161-170, 2012.

[26] P. B. Hedlund, P. E. Danielson, E. A. Thomas, K. Slanina, M. J. Carson, and J. G. Sutcliffe, "No hypothermic response to serotonin in 5- $\mathrm{HT}_{7}$ receptor knockout mice," Proceedings of the National Academy of Sciences of the United States of America, vol. 100, no. 3, pp. 1375-1380, 2003.

[27] T. Stein, N. Salomonis, and B. A. Gusterson, "Mammary gland involution as a multi-step process," Journal of Mammary Gland Biology and Neoplasia, vol. 12, no. 1, pp. 25-35, 2007.

[28] D. R. Pitelka, S. T. Hamamoto, J. G. Duafala, and M. K. Nemanic, "Cell contacts in the mouse mammary gland. I. Normal gland in postnatal development and the secretory cycle," Journal of Cell Biology, vol. 56, no. 3, pp. 797-818, 1973. 
[29] N. D. Horseman and R. J. Collier, "Serotonin: a local regulator in the mammary gland epithelium," Annual Review of Animal Biosciences, vol. 2, no. 1, pp. 353-374, 2014.

[30] A. M. Marshall, V. P. Pai, M. A. Sartor, and N. D. Horseman, "In vitro multipotent differentiation and barrier function of a human mammary epithelium," Cell and Tissue Research, vol. 335, no. 2, pp. 383-395, 2009.

[31] K. Lundgren, R. Montes de Oca Luna, Y. B. McNeill et al., "Targeted expression of MDM2 uncouples $S$ phase from mitosis and inhibits mammary gland development independent of p53," Genes and Development, vol. 11, no. 6, pp. 714-725, 1997.

[32] K. Hosokawa, D. Aharoni, A. Dantes et al., "Modulation of Mdm2 expression and p53-induced apoptosis in immortalized human ovarian granulosa cells," Endocrinology, vol. 139, no. 11, pp. 4688-4700, 1998.

[33] C. J. Foster and G. Lozano, "Loss of p19ARF enhances the defects of $\mathrm{Mdm} 2$ overexpression in the mammary gland," Oncogene, vol. 21, no. 22, pp. 3525-3531, 2002.

[34] A. Agrawal, J. Yang, R. F. Murphy, and D. K. Agrawal, "Regulation of the p14ARF-Mdm2-p53 pathway: an overview in breast cancer," Experimental and Molecular Pathology, vol. 81, no. 2, pp. 115-122, 2006.

[35] C. Sol, J. H. Jang, M. O. Riener et al., "Serotonin promotes tumor growth in human hepatocellular cancer," Hepatology, vol. 51, no. 4, pp. 1244-1254, 2010.

[36] V. S. Tharayil, M. M. Wouters, J. E. Stanich et al., "Lack of serotonin $5-\mathrm{HT}_{2 B}$ receptor alters proliferation and network volume of interstitial cells of Cajal in vivo," Neurogastroenterology and Motility, vol. 22, no. 4, pp. e462-e110, 2010. 

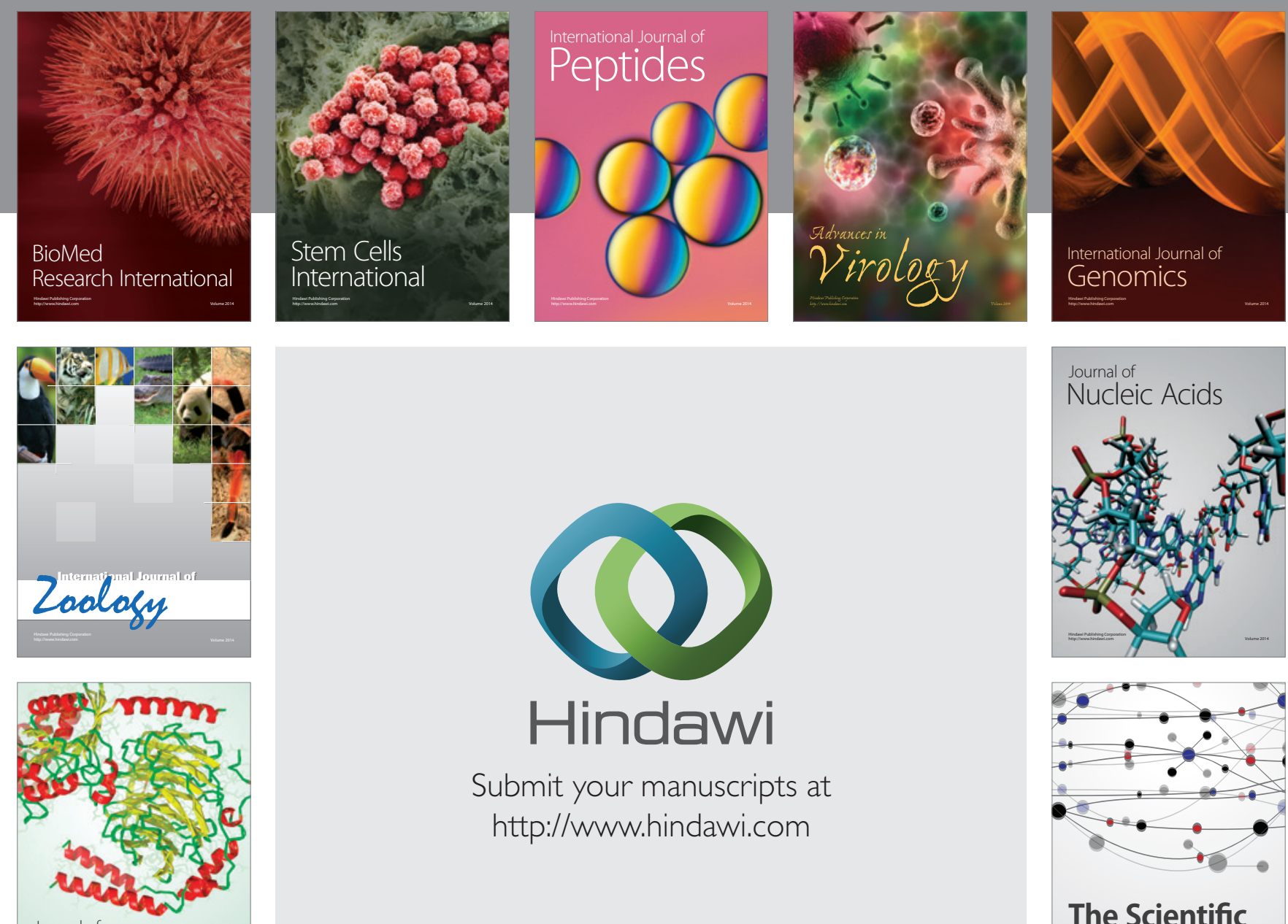

Submit your manuscripts at

http://www.hindawi.com

Journal of
Signal Transduction
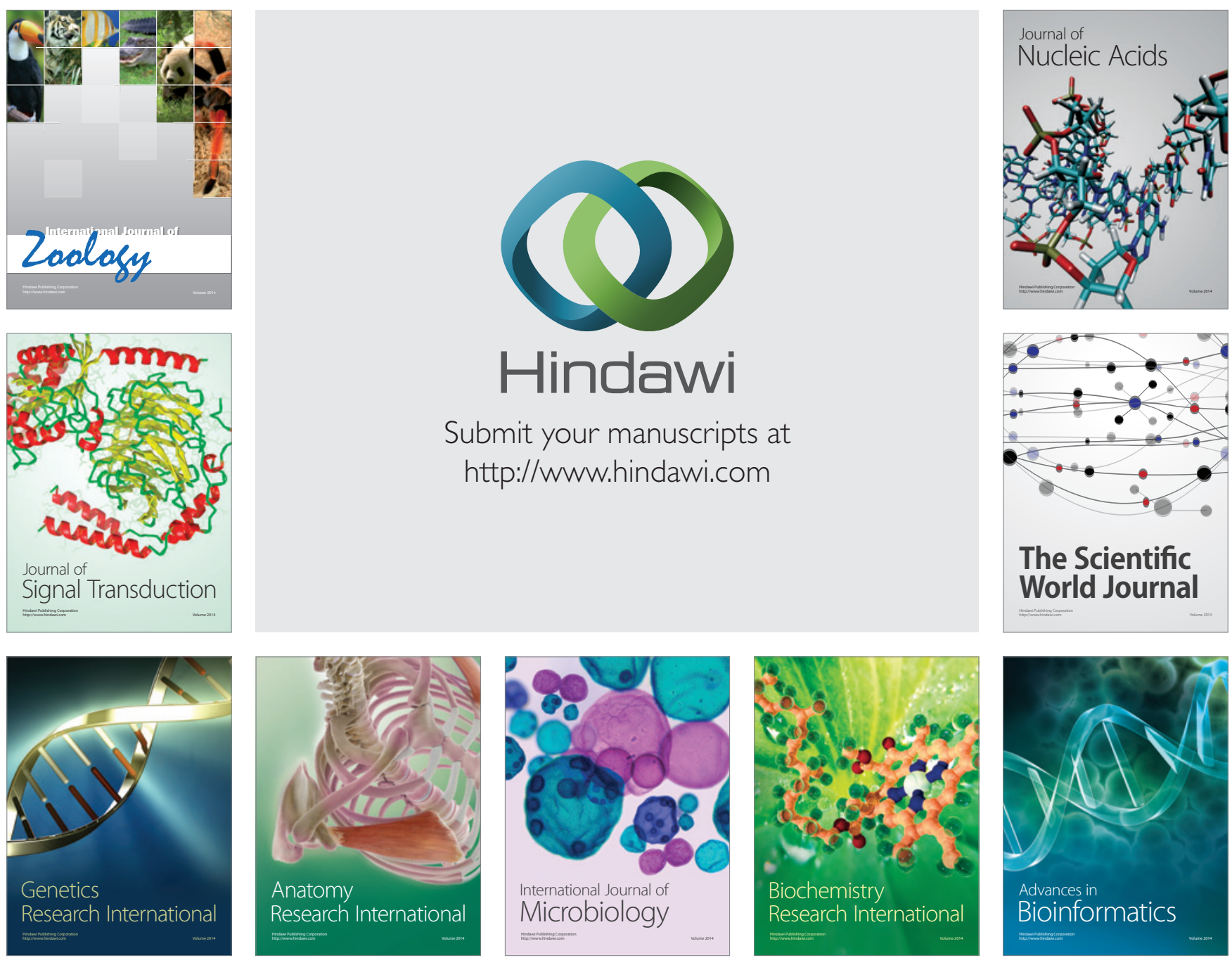

The Scientific World Journal
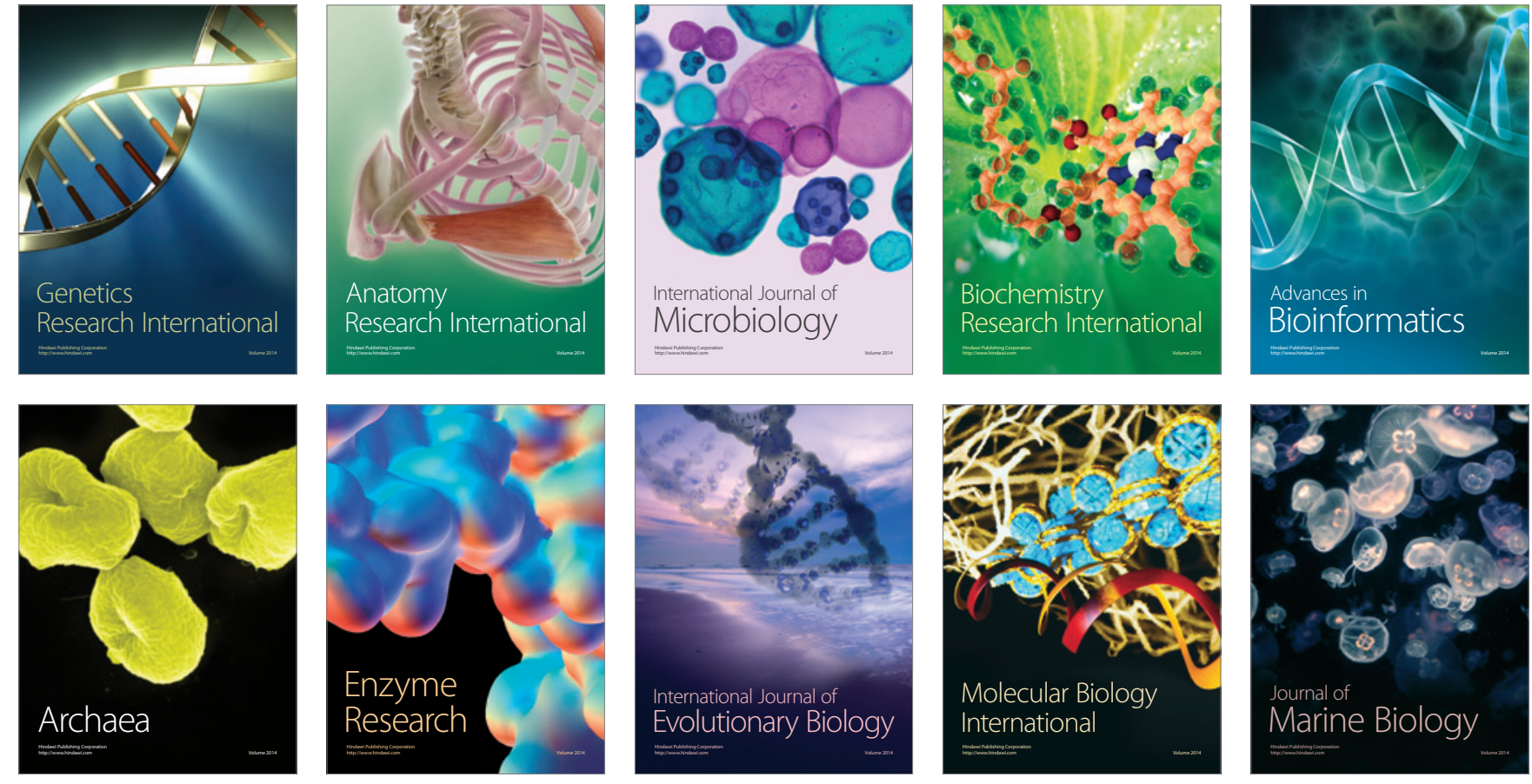